\title{
Conditional Probabilistic Population Projections: An Application to Climate Change
}

Brian C. O’Neill (oneill@iiasa.ac.at)

\section{Approved by}

Wolfgang Lutz (lutz@iiasa.ac.at)

Leader, Population Project

October 20, 2003 Institute, its National Member Organizations, or other organizations supporting the work. 


\section{Contents}

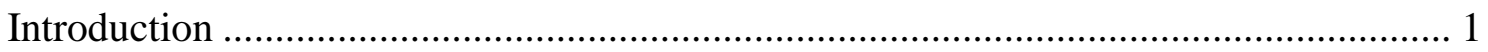

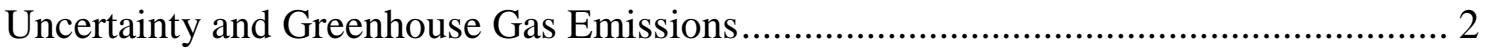

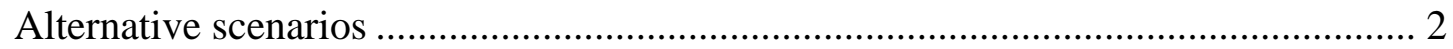

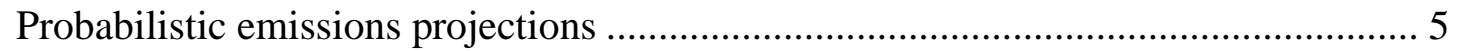

Conditional probabilistic emissions projections: Rationale ..................................... 6

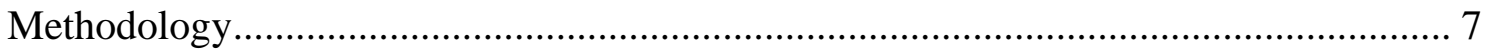

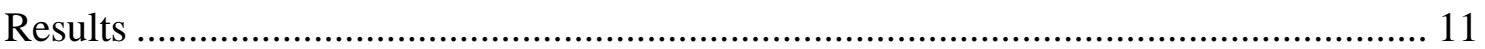

Conditional probabilistic population projections .................................................. 11

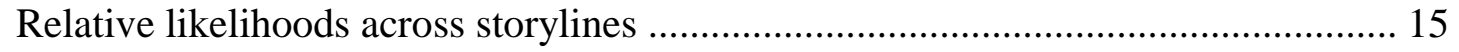

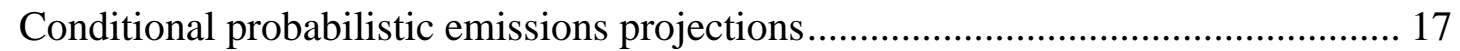

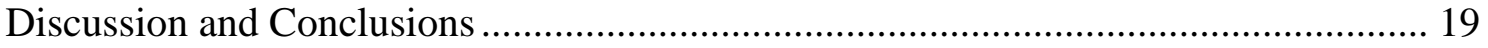

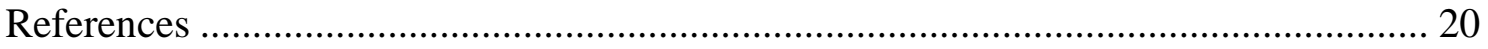




\section{Abstract}

Future changes in population size, composition, and spatial distribution are key factors in the analysis of climate change, and their future evolution is highly uncertain. In climate change analyses, population uncertainty has traditionally been accounted for by using alternative scenarios spanning a range of outcomes. This paper illustrates how conditional probabilistic projections offer a means of combining probabilistic approaches with the scenario-based approach typically employed in the development of greenhouse gas emissions projections. The illustration combines a set of emissions scenarios developed by the Intergovernmental Panel on Climate Change (IPCC) with existing probabilistic population projections from IIASA. Results demonstrate that conditional probabilistic projections have the potential to account more fully for uncertainty in emissions within conditional storylines about future development patterns, to provide a context for judging the consistency of individual scenarios with a given storyline, and to provide insight into relative likelihoods across storylines, at least from a demographic perspective. They may also serve as a step toward more comprehensive quantification of uncertainty in emissions projections. 


\section{Acknowledgments}

This paper has been submitted to the International Statistical Review for publication. An earlier version was presented at a workshop on "How to deal with uncertainty in population forecasting" held at the Vienna Institute of Demography, December 12-14, 2002, Vienna, Austria.

The author thanks Wolfgang Lutz and Sergei Scherbov for many valuable discussions, and Emilio Moran for helpful suggestions on clarifying the text. 


\title{
Conditional Probabilistic Population Projections: An Application to Climate Change
}

\author{
Brian C. O’Neill
}

\section{Introduction}

The threat of human-induced climate change, popularly known as global warming, presents a difficult challenge to society over the coming decades (Watson et al. 2001). The production of so-called "greenhouse gases" as a result of human activity, mainly due to energy production through the burning of fossil fuels such as coal, oil, and natural gas, is expected to lead to a generalized warming of the Earth's surface, rising sea levels, and changes in precipitation patterns. The potential impacts of these changes are many and varied - more frequent and intense heat waves, changes in the frequency of droughts and floods, increased coastal flooding, and more damaging storm surges all with attendant consequences for human health, agriculture, economic activity, biodiversity, and ecosystem functioning.

Future changes in population size, composition, and spatial distribution are key factors in the analysis of climate change (O'Neill et al. 2001). Demographics enter the problem in a number of ways: as one determinant (among many others) of emissions of greenhouse gases, and therefore of how much climate may change in the future; as a determinant of the impacts of climate change, and therefore how serious any given level of climate change might be; and potentially as a factor in political arrangements for addressing climate change (as, for example, in emissions reduction schemes based on per capita emissions levels). It is not just population size that matters: age structure, urbanization, and composition by household size and type are all of potential importance to these different aspects of the climate issue, as are population characteristics that are less demographic in the traditional sense but are often analyzed by demographers: educational status, health status, and poverty.

As demographers are well aware, there is great uncertainty in projecting any of these factors into the long-term future. However, the population component of the climate problem is in no way unusual in this respect. Uncertainties can be even larger in other factors, such as future labor productivity, rates of technological progress and of per capita GDP growth, consumption patterns, the response of the climate system to greenhouse gas emissions, and impacts of climate change on ecosystems and societies.

Recently, growing attention has been focused on methods of accounting for uncertainty in various components of the climate problem (see, e.g., Webster et al. 2002; Wigley and Raper 2001; Moss and Schneider 2000; Lempert et al. 2000; Nordhaus and Popp 1997). However, no study has devoted any specific attention to quantification of population uncertainty and its relevance to integrated assessments of 
climate change, despite the existence of fully probabilistic, global population projections (Lutz et al. 2001).

This paper proposes a new approach to accounting for demographic uncertainty within projections of greenhouse gas emissions. Accounting for uncertainty in emissions is important for at least two reasons. First, emissions projections play a key role in estimating what the consequences of climate change might be if no action were taken in response to it. Such estimates essentially define climate change as a problem to the extent that serious consequences seem possible. Of course other uncertainties, e.g., in the response of the climate system to emissions, and the impacts of climate change on society and ecosystems, are important as well. But emissions, as the first step in this "cascade of uncertainty" (Moss and Schneider 2000), play a key role. Second, analyses of the costs of achieving given emissions reduction targets are sensitive to the so-called reference emissions path: the assumed level of emissions over time in the absence of policy. Reaching a given target in a high-emissions world would be much more difficult than in a world in which emissions would turn out to be quite low anyway.

To date, there have been two main approaches to accounting for uncertainty in emissions: alternative scenarios and fully probabilistic projections. Here an approach is proposed that combines elements of both: conditional probabilistic projections. The next section describes current approaches in more detail, and the rationale for the conditional probabilistic approach. Following that, the methodology is discussed and applied to a well-known set of emissions scenarios. Results are presented, and the paper concludes with a short discussion.

\section{Uncertainty and Greenhouse Gas Emissions}

\section{Alternative scenarios}

Currently, the most prominent estimate of a plausible range for climate change consequences in the absence of climate policy is the one developed by the Intergovernmental Panel on Climate Change (IPCC) as part of its most recent assessment of climate change (Cubasch et al. 2001). Based on a set of 35 different emissions scenarios, combined with models that represent current estimates of uncertainty in greenhouse gas cycles and climate responses, the IPCC estimates that the increase in global average temperature over the next 100 years could range from $1.4 \mathrm{C}$ to $5.8 \mathrm{C}$ in the absence of climate policy.

The 35 emissions scenarios on which the IPCC projections of future climate change are based (see the IPCC Special Report on Emissions Scenarios (SRES), Nakicenovic et al. 2000) are explicitly not probabilistic. Instead, the rationale was to explore a wide range of plausible futures, each "equally sound" (Nakicenovic et al. 2000) but with no judgment made as to their relative likelihood. The development and analysis of alternative scenarios as an approach to uncertainty has a history of at least 50 years, beginning with post-World War II military planning, extending to business strategy development for major corporations, and more recently to planning for sustainable development (Schwartz 1991). Scenarios are stories about the way the future might unfold. They can be qualitative, quantitative, or both, and they can be used in a variety of ways, including for educating participants in the scenario development 
process about the issues at hand, or for communicating key insights to the intended audience. Scenarios tend to be used as an approach to uncertainty when problems are complex and uncertainties are very large, precluding meaningful estimates of the likelihood of various future outcomes. In decision analysis, scenarios are often used as a basis for searching for "robust strategies," i.e., options for responding to a problem that are likely to work reasonably well regardless of how the future unfolds (e.g., Lempert et al. 2000). Having a sufficiently diverse set of scenarios is a prerequisite for this type of analysis.

The SRES scenario development process consisted of three main steps. First, four different qualitative "storylines" were developed, describing broad socioeconomic and technological development patterns that could unfold over the $21^{\text {st }}$ century. While the SRES storylines are multidimensional, they can usefully be distinguished along two dimensions, "globalization" and "sustainability." Globalization refers to "the extent of economic convergence and social and cultural interactions across the regions" and sustainability to "the balance between economic objectives and environmental and equity objectives" (Nakicenovic et al. 2000, Section 4.2.1). The storylines were called A1, A2, B1, and B2, with no significance given to this ordering; 1 vs. 2 distinguishes the degree of globalization vs. regionalization, and the A vs. B distinction is based on the degree of economic vs. environmental orientation. Brief summaries are included in Table 1 to make the discussion more concrete.

Next, particular quantitative paths for fundamental driving forces of emissions, including population and gross domestic product (GDP), were selected that were judged to be consistent with each storyline. Finally, six different modeling teams produced quantitative interpretations of the storylines, using the quantitative paths for driving forces as inputs, resulting in 40 different scenarios for energy use, land use, and associated greenhouse gas emissions over the next 100 years. Figure 1 shows the range of $\mathrm{CO}_{2}$ emissions resulting from the SRES scenarios, categorized by storyline. The range is very wide, covering a possible increase in emissions of $\mathrm{CO}_{2}$ by a factor of 5 over the next 100 years, as well as a decline to emissions below today's level. 
Table 1. Brief summaries of the SRES storylines, from Nakicenovic et al. (2000, Section 4.2.1).

\section{Storyline Summary}

"The A1 storyline and scenario family describes a future world of very rapid economic growth, low population growth, and the rapid introduction of new and more efficient technologies. Major underlying themes are convergence among regions, capacity building, and increased cultural and social interactions, with a substantial reduction in regional differences in per capita income. The A1 scenario family develops into four groups that describe alternative directions of technological change in the energy system."

A2 "The A2 storyline and scenario family describes a very heterogeneous world. The underlying theme is self-reliance and preservation of local identities. Fertility patterns across regions converge very slowly, which results in high population growth. Economic development is primarily regionally oriented and per capita economic growth and technological change are more fragmented and slower than in other storylines."

B1 "The B1 storyline and scenario family describes a convergent world with the same low population growth as in the A1 storyline, but with rapid changes in economic structures toward a service and information economy, with reductions in material intensity, and the introduction of clean and resource-efficient technologies. The emphasis is on global solutions to economic, social, and environmental sustainability, including improved equity, but without additional climate initiatives."

B2 "The B2 storyline and scenario family describes a world in which the emphasis is on local solutions to economic, social, and environmental sustainability. It is a world with moderate population growth, intermediate levels of economic development, and less rapid and more diverse technological change than in the B1 and A1 storylines. While the scenario is also oriented toward environmental protection and social equity, it focuses on local and regional levels." 
Figure 1. Projections of global $\mathrm{CO}_{2}$ emissions from fossil fuel for each SRES storyline and variant. Source: Nakicenovic et al. (2000).

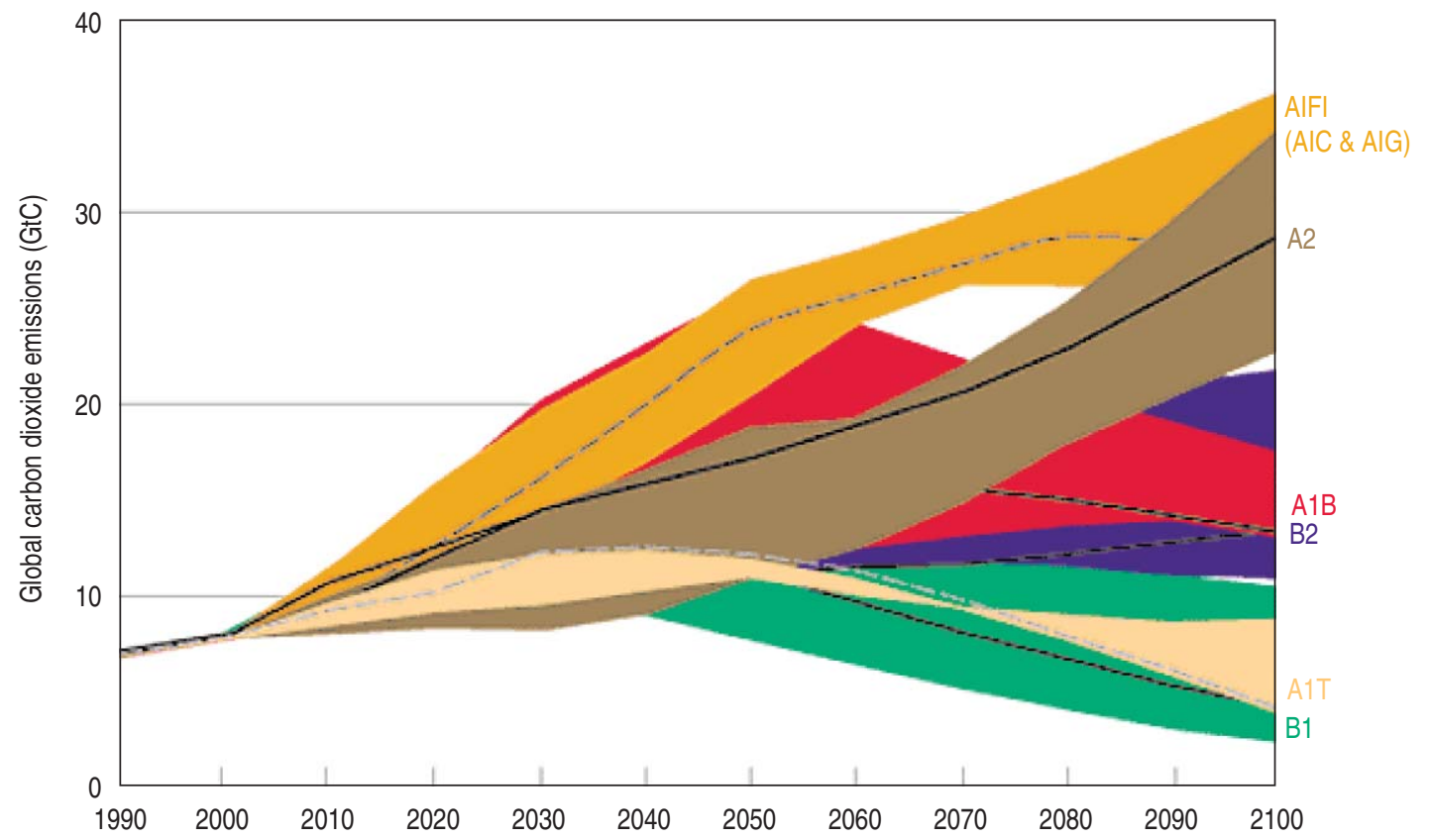

\section{Probabilistic emissions projections}

Several studies have produced fully probabilistic emissions projections. Early work by Nordhaus and Yohe (1983) and Edmonds et al. (1986) developed probabilistic versions of energy-economic growth models to calculate uncertainty distributions for optimal emissions, given uncertainty in a long list of parameters, including those affecting population growth, labor productivity, the energy system (e.g., elasticities of substitution between fuels), the carbon cycle, and climate response. These projections were the first to systematically explore uncertainty in long-term emissions projections, and were useful in estimating how wide the plausible range of outcomes actually was (Keepin 1986).

More recently, Wigley and Raper (2001) made their own assumptions about the relative likelihood of the SRES scenarios. They assign each SRES scenario an equal probability as part of an effort to produce probabilistic projections of global average temperature change (see also New and Hulme (2000) and Dessai and Hulme (2001) for similar approaches). In contrast, Webster et al. (2002) take an approach independent of SRES. They use an expert opinion-based estimate of uncertainty distributions for key parameters of an energy-economic growth model, which they then use to generate an uncertainty distribution for future emissions and climate change.

There are a number of points of view on the desirability, and credibility, of assigning subjective relative probabilities to alternative scenarios of future emissions. Schneider (2001, 2002) argues for developing subjective probability estimates based on expert opinion, since the judgment of experts is preferable, in his view, to the plethora 
of judgments that different users and policy makers will make in the absence of guidance on this point. Webster et al. (2002) amplify this view, describing the question as not one of whether probabilities will be assigned to scenarios, but when and by whom. Since methods have been developed to control known biases in expert judgment, it is better that probabilities be assigned by experts in decision analytic techniques than by users. In contrast, Grübler and Nakicenovic (2001) argue that assigning subjective probabilities to emissions scenarios would be inappropriate and infeasible. Probability distributions for conditions such as population, economic growth, technological development, agricultural practices, diets, etc. (all determinants of emissions) would themselves be insufficient; probabilities for ways in which these conditions would interact would also be necessary, producing an unmanageable explosion of uncertainty, in their judgment. Attempts to assign subjective probabilities in the face of such complications risks "dismissal of uncertainty in favour of spuriously constructed 'expert' opinion."

\section{Conditional probabilistic emissions projections: Rationale}

One route to managing the complexity of the problem is to preserve the scenario approach to uncertainty when considering possible broad patterns of development in the future (i.e., storylines), but use subjective probabilities in quantifying uncertainty in the consequences of those development patterns for emissions. The basic rationale for such conditional probabilistic projections is that more meaningful judgments can be made about the likelihood of future trends given a particular development path, than can be made about the relative likelihood of different development paths actually occurring. Stated in terms of the SRES scenarios, one may be able to assign more meaningful probabilities to emissions outcomes conditional on, say, the A2 world coming to pass, than one could to the relative likelihood of the A1, A2, B1 and B2 storylines occurring in the first place.

Intuitively, one expects this proposition would hold, because by reducing the state space that must be considered, the conditional approach makes uncertainties more manageable. For example, it may be judged difficult, if not impossible, to assign meaningful probabilities to the rate of per capita economic growth over the coming decades. One reason may be that it is difficult to judge how globalization is likely to proceed - whether trade barriers will become more or less restrictive, for example which will have important consequences for economic growth. However, if one restricts the set of possible futures that must be considered to only those in which globalization proceeds rapidly and trade barriers are reduced, confidence in judgments of the probability distribution of future economic growth rates, conditional on that storyline, would increase. This type of reasoning can be extended to many different factors that are included in storylines of the future. In fact, it would seem that as long as the conditions placed on the future (through storylines) are relevant to the outcomes of interest, then it should be possible to make better judgments of conditional probabilities of outcomes than of unconditional ones.

The following sections illustrate the possibility of developing conditional probabilistic emissions scenarios, using the IPCC SRES scenarios as a basis. First, probabilistic population projections conditional on the storylines used in the SRES scenarios are developed. Next, they are combined through simple linear scaling, with 
per capita emissions rates derived from the SRES scenarios. While this latter step is an obvious oversimplification of the relationships between population and other factors affecting emissions, it serves to illustrate the kind of emissions results one might obtain from a fuller accounting for population uncertainty.

The results of developing conditional probabilistic projections for both population and for emissions can be useful for several reasons:

1. They can be used as a means of judging the likelihood of a single existing scenario (of population or of emissions) given a particular storyline;

2. They can be used to develop a single new scenario that has a probabilistic interpretation for use in scenario analysis (e.g., O'Neill et al. forthcoming);

3. They can provide a fuller accounting for uncertainty (in population or in emissions) within storylines;

4. They might provide some insight into the relative likelihood of outcomes across storylines (discussed further below);

5. They may serve as a step toward more comprehensive probabilistic projections in the future.

\section{Methodology}

Conditional probabilistic population forecasts were first discussed by Alho (1997), who began with known unconditional and conditional projections, and evaluated the plausibility that the conditional projections could be derived from the unconditional ones. Sanderson et al. (forthcoming and 2003) take a different approach, beginning with unconditional projections and deriving conditional forecasts that demonstrate, within a probabilistic framework, the sensitivity of outcomes to key variables. Lutz and Scherbov (2002) use the conditional probabilistic approach in a similar manner to compare the sensitivity of the European population to changes in immigration and fertility. These studies demonstrate that sensitivity analysis, traditionally carried out within a deterministic framework, is also possible within a probabilistic framework. This paper builds on that approach by applying the concept of conditional probabilistic projections to emissions scenario development. The necessary steps are:

1. Specify qualitative descriptions of future trends in demographic rates (fertility, mortality, migration) judged to be consistent with a given storyline;

2. Define quantitative probability distributions of demographic rates consistent with the qualitative descriptions;

3. Generate a probability distribution of population outcomes conditional on the distributions of demographic rates;

4. Generate a probability distribution of emissions based on the distribution of population outcomes.

Table 2 provides a summary of the qualitative fertility, mortality and migration assumptions made by the SRES authors for each storyline for the industrialized country regions (IND) and the developing country regions (DEV). The "high," "medium," and "low" descriptions are interpreted as relative to the overall outlook within each region (i.e., high fertility in the IND region means the high end of the plausible range for that 
region, but may in fact be lower than low fertility paths for the DEV region, which occupy the low end of the plausible range for the DEV region). Based on descriptions in the SRES report (primarily in Sections 4.3 and 4.4), a summary of the reasoning for these choices is as follows:

- In the A1 storyline, rapid economic development, associated with improved education and reduced income disparities, is assumed to drive a relatively rapid fertility decline in the high fertility regions. Fertility is generally below replacement level in the long run. Fertility in industrialized regions is assumed to follow a medium path at least in part so that, relative to the developing regions, the scenario "is consistent with the assumption of convergence of social and economic development" (p. 193); i.e., the assumption is that social and economic convergence will lead to demographic convergence as well. For mortality, it is assumed that the conditions leading to low fertility are also consistent with relatively low mortality, so mortality is assumed to be low in all regions. No explicit discussion of migration is provided, although the projection eventually adopted assumes medium migration levels.

- In the A2 storyline, the regional orientation and slower rate of economic growth, limited flow of people and ideas across regions, and orientation toward family and community values was judged to be consistent with a relatively high fertility in all world regions. Mortality was assumed to be high as well, based on the assumption that conditions leading to high fertility would also lead to relatively high mortality in all regions. Although the storyline describes a limited flow of people across regions, the medium migration flows were chosen, as in all other storylines.

- The B1 storyline shares the same population projection as the A1 storyline, although for somewhat different reasons. Rapid social development, particularly for women, and an emphasis on education drives a relatively rapid decline in fertility in developing country regions (as opposed to the A1 storyline, in which economic development is seen as the main driver). Reasoning for fertility in industrialized countries, and for mortality and migration assumptions, are the same as in A1.

- In the B2 storyline, economic development is moderate, particularly in the developing country regions. However education and welfare programs are pursued widely and local inequity is reduced through strong community support networks. The mix of moderate economic development, and strong but heterogeneous social development results in an assumption of medium fertility and mortality paths. Migration is again assumed to be medium, with no explicit discussion of this choice.

For each storyline, the SRES authors selected one population projection from the literature that was judged to be consistent with these assumptions (see Table 2). A similar approach (selection or creation of a single scenario within storylines) was taken for quantifying GDP and rates of technological progress within storylines.

The next step in developing conditional probabilistic projections to substitute for the single scenarios used in SRES is to express the qualitative demographic assumptions associated with each storyline as quantitative uncertainty distributions. These 
conditional distributions are derived from the global probabilistic projection from IIASA (Lutz et al. 2001). The methodology for generating the IIASA projection consists of a combination of expert- and argument-based judgment on future vital rates (fertility, mortality, and migration), a time series approach to generating individual trajectories for each vital rate with realistic autocorrelation, and consideration of the magnitude of errors in past projections to guard against underestimation of uncertainty. The final product is a distribution of 2000 equally likely simulations at the level of 13 world regions. The conditional probabilistic projections are generated by selecting from this complete (unconditional) distribution a subset of simulations consistent with the SRES assumptions as expressed in Table 2.

Table 2. Demographic assumptions in SRES storylines (IND = industrialized country regions; $\mathrm{DEV}=$ developing country regions).

\begin{tabular}{lllll}
\hline Storyline & Fertility & Mortality & Migration & Projection Source \\
\hline A1/B1 & IND: medium & IND: low & IND: medium & Lutz et al. 1996 \\
& DEV: low & DEV: low & IND: medium & \\
A2 & IND: high & IND: high & IND: medium & Lutz et al. 1996 \\
& DEV: high & DEV: high & IND: medium & \\
B2 & IND: medium & IND: medium & IND: medium & UN 1998 \\
& DEV: medium & DEV: medium & IND: medium & \\
\hline
\end{tabular}

In principle, the "high," "medium," and "low" categories for demographic rates would be best defined by the scenario-builders themselves, because they may have had specific ideas in mind that are not clearly stated in the storyline descriptions. For example, was "high" fertility in the A2 world intended to imply a rather extreme case (perhaps near the $95^{\text {th }}$ percentile of possible outcomes), or simply a fertility rate that is moderately higher than an unconditional medium or "best guess" outcome? Should fertility always be high in this scenario, or should it be high on average, with periods of medium or low fertility also possible? Should it be high in all regions simultaneously, or high on average across all regions? In the absence of specific guidance in the SRES report, this paper takes as a starting point that high, medium, and low should represent ranges of demographic rates that are equally likely and are mutually exclusive. That is, in each region and for each variable representing one of the demographic components

\footnotetext{
${ }^{1}$ It would be possible to offer alternative interpretations of the demographic rates most likely to be associated with each storyline, but it is considered outside the scope of this paper. For example, the rationale presented in SRES for associations between fertility and mortality, and between fertility, income growth, and social conditions, is based on experience in high fertility countries. However, it is built into the assumptions for the entire century and in some cases for all regions, even though during most of this period most regions will have completed the demographic transition (and despite the fact that the SRES report itself notes that while there may be a weak population-economic growth relationship in developing regions, the reverse may be true in higher income countries; see p. 113). In addition, migration assumptions are not varied across storylines, although the storylines appear to support different migration levels.
} 
(total fertility rate, life expectancy, and net migration) high, medium, and low should be defined as those ranges covering the top, middle, and bottom third, respectively, of all outcomes in the IIASA unconditional probabilistic projections.

To carry out the selection process, an index of each demographic rate must be adopted, and an appropriate level of regional aggregation chosen. As an index, the average values of each variable over the period 2000-2050 were used, since several essential elements of the reasoning behind the choice of population scenario in SRES apply to the currently high fertility regions (e.g., relationships between fertility and economic and social conditions, and the correlation between fertility and mortality), and these regions will have above replacement fertility mainly in the first half of the century. For regional disaggregation, average values for two world regions, Industrialized (IND) and Developing (DEV), were considered, which match the level of detail for which qualitative storyline elements relevant to demographics are provided in SRES. Averages are calculated from underlying rates in the IIASA projections. ${ }^{2}$

The selection process, therefore, consists of producing an average fertility, mortality, and migration rate for each of 1,000 simulations in the unconditional projection, with averaging performed over the period 2000-2050 and over two groups of regions. Simulations are then ranked according to each demographic rate in each region, and assigned to high, medium, and low categories by dividing each ranking into three sets of outcomes containing the first 333, next 334, and last 333 simulations. Those simulations matching the assumptions for all three rates for each SRES storyline as defined in Table 2 become part of the conditional probabilistic projection for that storyline. This approach produces what might be called input-constrained conditional probabilistic projections, since the conditions (storylines) impose constraints on the demographic rates that serve as inputs to the projection. ${ }^{3}$

Before examining results, it is important to stress that alternative approaches to the selection process are possible. For example, the definition of the high, medium, and low categories could be based on intervals of absolute values of demographic rates rather than on the assumption that each category should be equally likely. This strategy is not pursued here because there is no obvious basis for defining the intervals of absolute values that would clearly recommend it. Additionally, category definitions need not be derived by simply dividing the unconditional distributions into mutually exclusive terciles. This division is clearly a simplification that gives all simulations within a tercile a weight of 1 , and all outside the tercile a weight of zero. One could consider a definition in which a more smoothly varying distribution of weights was applied to the unconditional distribution. Furthermore, one could choose a different metric than the 50-year average value used here. For example, one might choose to extend the averaging period to 100 years, or to use consecutive or overlapping 50-year periods to extend the constraints over the whole century (O'Neill et al. forthcoming). These choices have implications for trends in the medians and for the variance of the

\footnotetext{
${ }^{2}$ Fertility, life expectancy, and net migration for the IND and DEV regions are specified as the population-weighted average of these variables across the subregions. For fertility, the population aged 15-65 is used, the most relevant age group available. For net migration, the absolute values of the subregional levels are used.

${ }^{3}$ It is also possible to develop output-constrained projections, in which constraints on population size or age structure are used to select individual simulations, a type of inverse approach.
} 
conditional distributions of the underlying rates. Finally, one could choose different levels of regional aggregation to which the criteria are applied. Applying criteria to all 13 IIASA regions simultaneously, versus applying them to averages across several regions, also affects the variance of the conditional distributions of demographic rates. The methodological choices made in this paper are intended to match the level of specificity provided in the SRES storylines, and to remain as simple as possible. Exploring alternatives is left to future work.

\section{Results}

First we discuss the results for the population outcomes, and then for emissions.

\section{Conditional probabilistic population projections}

Figures $2 \mathrm{a}-\mathrm{c}$ show the results of the process of selection of population distributions, for the world and two regions for the A2 storyline. Figure $2 \mathrm{a}$ shows the individual simulations underlying the conditional projection for the world, along with the median and $60 \%$ and $95 \%$ uncertainty intervals. For comparison, the single population path used in the SRES A2 storyline is shown as a red line. Two main conclusions emerge. First, the probabilistic projections indicate substantial scope for uncertainty in population size conditional on the A2 storyline. The $60 \%$ uncertainty interval for global population in 2100 is 8.5 to 12 billion, and the $95 \%$ uncertainty interval is 7 to 15 billion. Second, the SRES population assumption is high relative to the conditional probabilistic projection. Early in the century it falls at about the upper limit of the $60 \%$ uncertainty interval, and by the end of the century is at 15 billion, the upper limit of the $95 \%$ uncertainty interval and about 4 billion higher than the median.

The systematic downward bias in the probabilistic projections relative to the SRES assumptions for A2 is due primarily to changes in the outlook for fertility and mortality in different regions of the world. Figures $2 b$ and $2 c$ show that the DEV region mirrors the global result, with the SRES population size reaching the upper bound of the 95\% uncertainty interval by the end of the century, while the IND region assumption is high but more moderately so, falling just above the $60 \%$ uncertainty interval. More detailed regional analysis shows that difference between the SRES population assumptions and the conditional projections vary across smaller regions. Regions showing the largest differences include the Reforming Economies region (mainly the Former Soviet Union) and the Centrally Planned Asia region (mainly China) by the end of the century. 
Figure 2. Probabilistic population projections conditional on the A2 storyline. Gray lines: individual simulations making up the conditional probabilistic projections. Blue lines: median (solid), 60\% uncertainty interval (long dashed), and 95\% uncertainty interval (short dashed) for the IIASA 2001 conditional projections. Red line: SRES A2 population assumption. Figures a-c show results for the world, industrialized country region (IND), and developing country region (DEV), respectively.

(a)

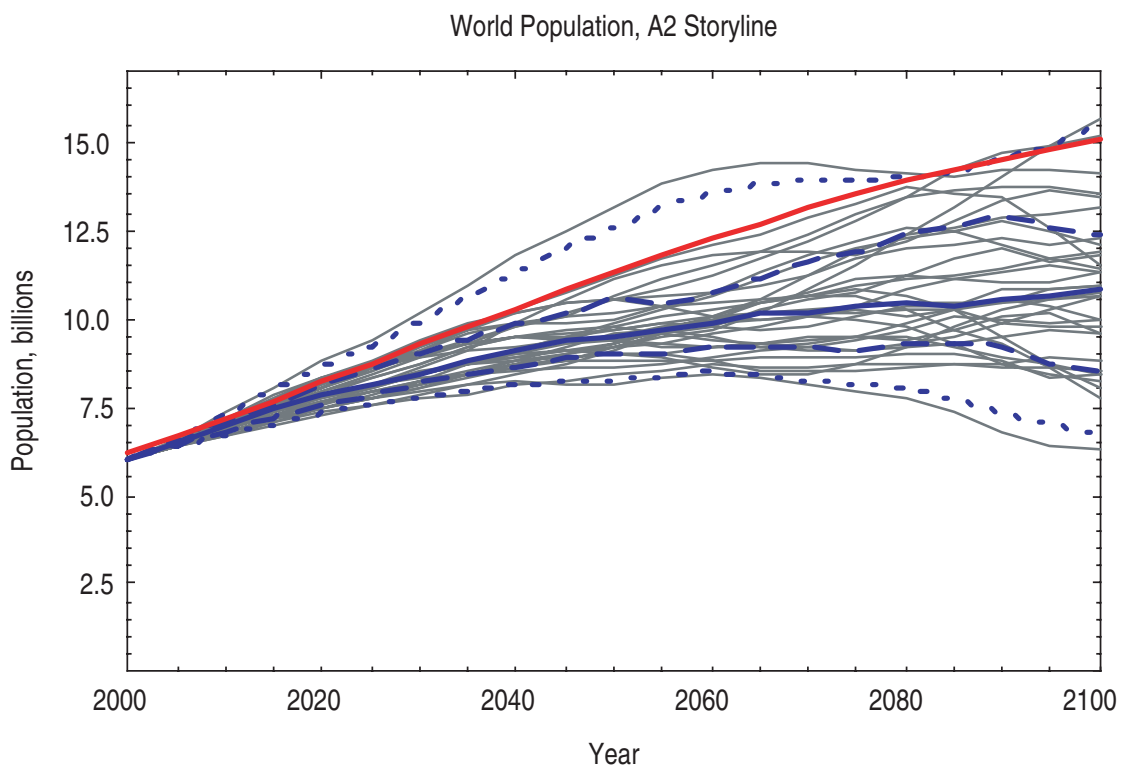

(b)

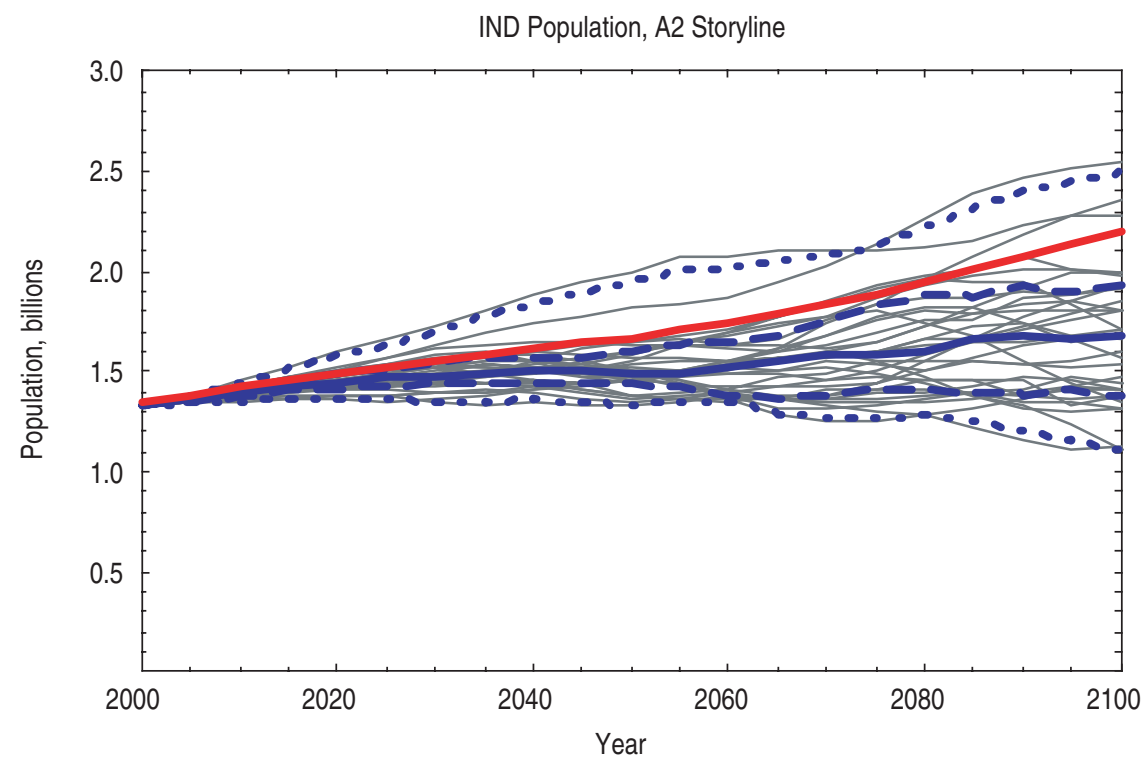


(c)

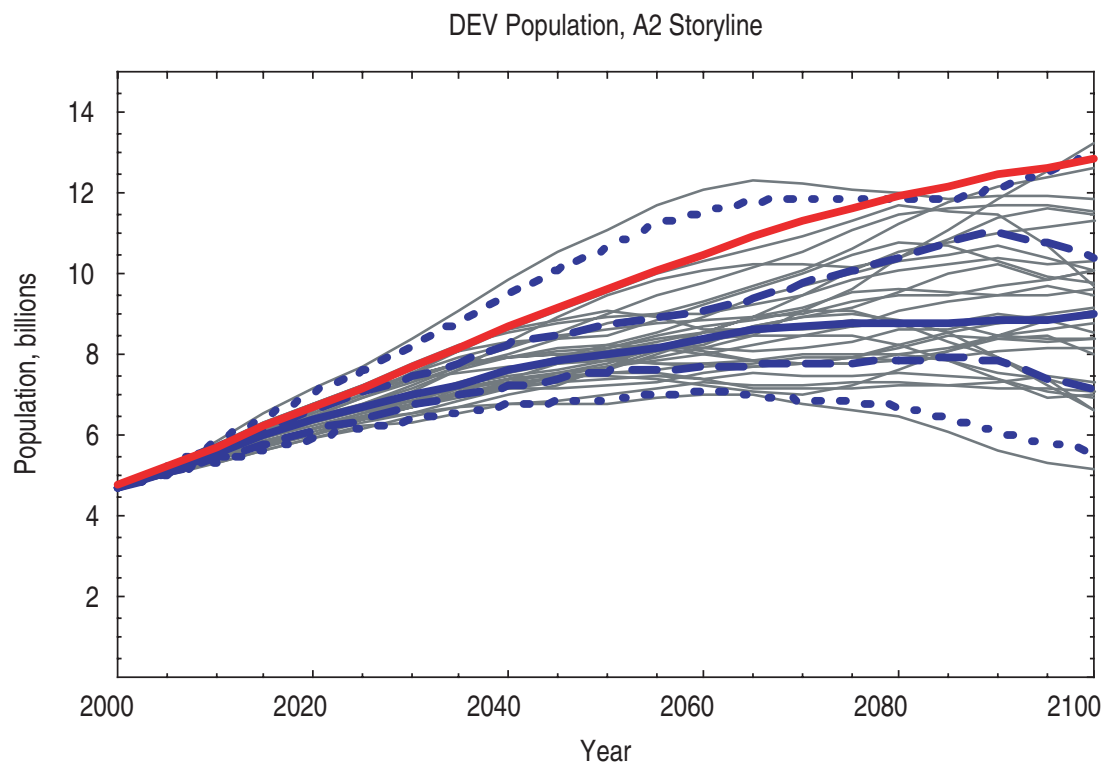

Figure 3 summarizes the results for all storylines for the years 2050 and 2100 for the world and the two regions. It shows that the uncertainty range in the conditional projections is largest in the A2 storyline (when fertility is assumed to be high), and the smallest in the A1/B1 storylines (when fertility is assumed to be low), although in all storylines the conditional projections show a 95\% uncertainty interval of at least $+/-20 \%$ in terms of absolute population size relative to the median. In the year 2050, the SRES population assumptions are relatively close to the median of the conditional projections for the A1/B1 and the B2 storylines. However by the end of the century, they appear high not only in the A2 storyline described above, but also in the DEV region in the B2 storyline (falling well above the $95 \%$ uncertainty interval), and in the IND region in the A1/B1 storyline (falling at about the upper end of the $95 \%$ uncertainty interval).

The quantitative differences between the SRES population assumptions and the conditional projections are sensitive to the definitions of high, medium, and low, as well as to the time periods and regional aggregations to which the criteria are applied, although the general patterns appear to be robust. 
Figure 3. Population size of the median and the bounds of the $60 \%$ and $95 \%$ uncertainty intervals in conditional probabilistic projections, relative to population size assumed in SRES, for the years 2050 (a) and 2100 (b).

(a)

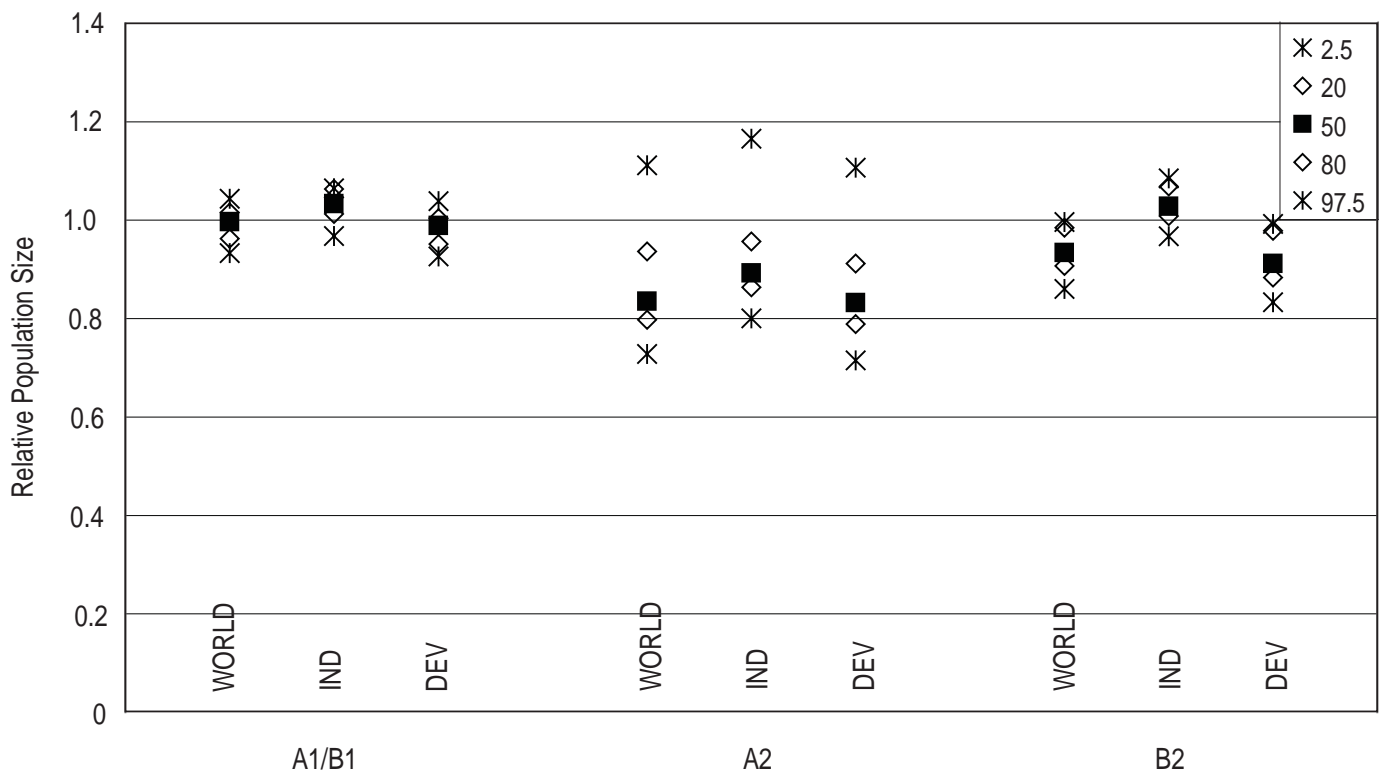

(b)

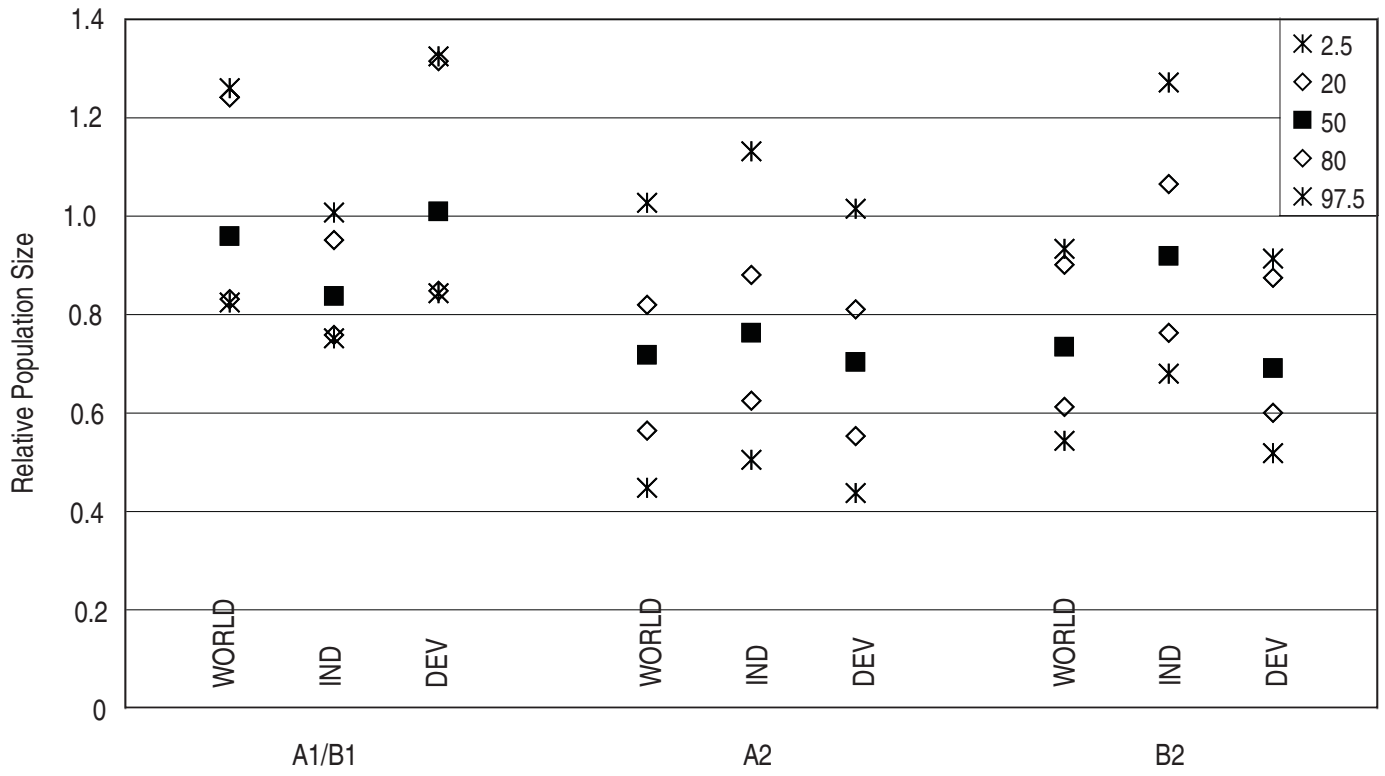




\section{Relative likelihoods across storylines}

One intriguing possibility offered by conditional probabilistic projections is that, if they are drawn from a full unconditional projection, they should provide a basis for judging the relative likelihood across two or more conditional projections, not just the relative likelihood of outcomes within a given conditional projection. For example, if a conditional projection based on storyline $\mathrm{X}$ consists of twice as many (equally likely) simulations drawn from the unconditional distribution as a conditional projection based on storyline $\mathrm{Y}$, then storyline $\mathrm{X}$ should be twice as likely as storyline $\mathrm{Y}$. Thus, comparing the conditional projections developed to be consistent with the four SRES storylines should give an indication of the relative likelihoods of the SRES storylines, at least from a demographic perspective. Such demographically-based relative likelihoods could be combined with perspectives on other aspects of the scenarios to inform a larger process of considering relative likelihoods of alternative emissions scenarios.

Table 3 shows, for each storyline, the number of simulations that match assumptions regarding each of the demographic rates individually, as well as combinations of rates. For example, it shows that, based on the definitions of high, medium, and low established above, there are 7 simulations (out of 1,000 total that were available for this analysis) consistent with the A1 and B1 storylines in terms of fertility, mortality, and migration at the level of two world regions. There are 32 simulations consistent with the A2 storyline, and 14 simulations consistent with the B2 storyline. Thus, given the IIASA population projections, the SRES storylines, and the category definitions used here, the demographic assumptions consistent with the B2 and especially the A1/B1 storylines appear to be substantially less likely than the assumptions underlying the A2 storyline.

Table 3. Number of simulations meeting fertility (F), life expectancy (LE), and migration (M) criteria, as well as combinations of them, associated with the SRES storylines.

\begin{tabular}{lccccc}
\hline Storyline & F & LE & M & F+LE & F+LE+M \\
\hline A1/B1 & 62 & 301 & 334 & 16 & 7 \\
A2 & 268 & 297 & 334 & 80 & 32 \\
B2 & 207 & 266 & 334 & 49 & 14 \\
\hline
\end{tabular}

There are two separate reasons for this result. The A1/B1 projection is relatively unlikely primarily because it mixes medium fertility rates in the IND region with low fertility rates in the DEV region. Because the IIASA projections assume a relatively strong correlation in fertility rates across regions, this combination is less likely than the A2 assumption of high fertility in both regions. As shown in Table 3, there are only 62 simulations that meet the A1/B1 fertility assumptions, while there are 268 simulations that meet the A2 fertility assumptions. 
The reason that the $\mathrm{B} 2$ projection is relatively unlikely is less obvious. A counterintuitive consequence of the regional correlations in demographic rates is that, even if it is equally likely that a single variable in a single region will fall in the high, medium, or low category at any given time, it is not equally likely that several regions will simultaneously experience high, medium, or low rates within one simulation. In general, it is substantially more likely that several regions will experience simultaneously low or high rates, than simultaneous medium rates. For example, Table 3 shows that while there are 268 simulations in which fertility is in the high category in each of the two world regions (i.e., in the A2 storyline), there are only 207 in which it is in the medium category in both regions (i.e., in the B2 storyline). This pattern holds for mortality assumptions as well: there are more simulations (297) in which mortality is high in both regions than simulations (266) in which it is medium in both regions. ${ }^{4}$

This result is likely due to a combination of factors. ${ }^{5}$ One factor is a consequence of the nature of the standard means of generating correlated variables with correlations measured by the correlation coefficient. This process weights extreme values more heavily (through the squared terms in the definition of the correlation coefficient) than values close to the means, and therefore the generation process produces values that are more strongly correlated when they are relatively high or low, and more weakly correlated near the means. Another factor is that, even if the correlation were identical across categories, imperfect correlation produces a relative deficit in pairs of medium values because the "medium" category is bounded both from above and from below, while the "high" category has only a lower boundary and the "low" category has only an upper boundary. As a result, when imperfect correlation spreads the location of variable pairs across the state space, more are "lost" from the medium category than from the high or low categories. The net result of both factors is that scenarios consistent with medium values for vital rates in all regions (such as B2) are less likely than scenarios consistent with high values for fertility and mortality in all regions (such as A2).

It is possible that this pattern could be consistent with expectations for the real world. It may be that extreme values of fertility or mortality in one region would be expected to exert a strong influence on rates in other regions, but that moderate values would have a weaker influence. However the empirical basis appears to be too thin to strongly support any particular claim at this point. Thus no great weight can be placed on conclusions regarding relative likelihoods of different storylines.

\footnotetext{
${ }^{4}$ Results follow the same general pattern when vital rates in particular years, rather than averaged over time, are used, and when based on alternative regions or groups of regions. In both cases, the differences in the likelihood of simultaneous high, medium, or low values is more extreme.

${ }^{5}$ Thanks to Wolfgang Lutz and Sergei Scherbov for these suggestions.
} 


\section{Conditional probabilistic emissions projections}

In principle, deriving conditional probabilistic emissions projections would require quantifying uncertainty within storylines not only in population, but also in each of the other elements driving emissions, as well as in the relationships between them. In this paper, the matter is greatly simplified by assuming that all SRES emissions scenarios within each storyline are equally likely, converting each to a per capita $\mathrm{CO}_{2}$ emissions path (excluding land use emissions), and combining all per capita emissions paths with all population simulations underlying the conditional population projections for each storyline. The result is a set of equally likely emissions paths for each storyline, which can then be interpreted as a conditional probabilistic emissions projections and compared with the scenarios developed in SRES.

Neither the assumption of equal likelihood across the SRES per capita emissions scenarios within storylines, nor the assumption of a linear relationship between population and per capita emissions, is well founded. As discussed above, the SRES authors made no judgments about relative likelihoods across scenarios, and attempts to make such judgments have been controversial. Here, the assumption of equal likelihood of emissions scenarios within storylines probably has more to recommend it than judgments across storylines, but still must be treated as speculative. Whether population is linearly related to per capita $\mathrm{CO}_{2}$ emissions or not is also questionable. There is some evidence that historically this has been the case (Dietz and Rosa 1997), and typical optimal growth models used in climate analysis also tend to demonstrate an essentially linear relationship (Gaffin and O'Neill 1997; O'Neill et al. 2001). However the dependence of emissions on population has not been analyzed in more detailed models.

Figure 4 shows the conditional probabilistic emissions projection for the A2 storyline, compared with the four A2 emissions scenarios from SRES. ${ }^{6}$ The same basic conclusions drawn for the conditional population projections hold here as well. First, the conditional probabilistic emissions projections indicate a substantially wider range of uncertainty in emissions than do the original four SRES scenarios. The $60 \%$ uncertainty interval is $18-27 \mathrm{GtC} / \mathrm{yr}$ by 2100 , and the $95 \%$ uncertainty interval is $14-32 \mathrm{GtC} / \mathrm{yr}$. In comparison, the SRES A2 scenarios span a much smaller range of 28-33 GtC/yr. Second, the probabilistic projections are generally substantially lower than the SRES A2 emissions scenarios at the global level. While one of the four SRES scenarios is below the median over the first 30 years, by the end of the century all four are above the $60 \%$ uncertainty interval, and one of them is above the $95 \%$ uncertainty interval.

\footnotetext{
${ }^{6}$ Two SRES scenarios were dropped from this storyline: A2 IMAGE, results of which are not provided in SRES for all time periods, and A2-A1 MINICAM which models a combined storyline scenario that switches to A1 assumptions partway through the simulation period. The conditional projections therefore consist of 128 simulations ( $32 \times 4)$.
} 
Figure 4. Probabilistic emissions projections for the world conditional on the A2 storyline. Blue lines: median (solid), 60\% uncertainty interval (long dashed), and 95\% uncertainty interval (short dashed). Red line: SRES A2 emission scenarios.

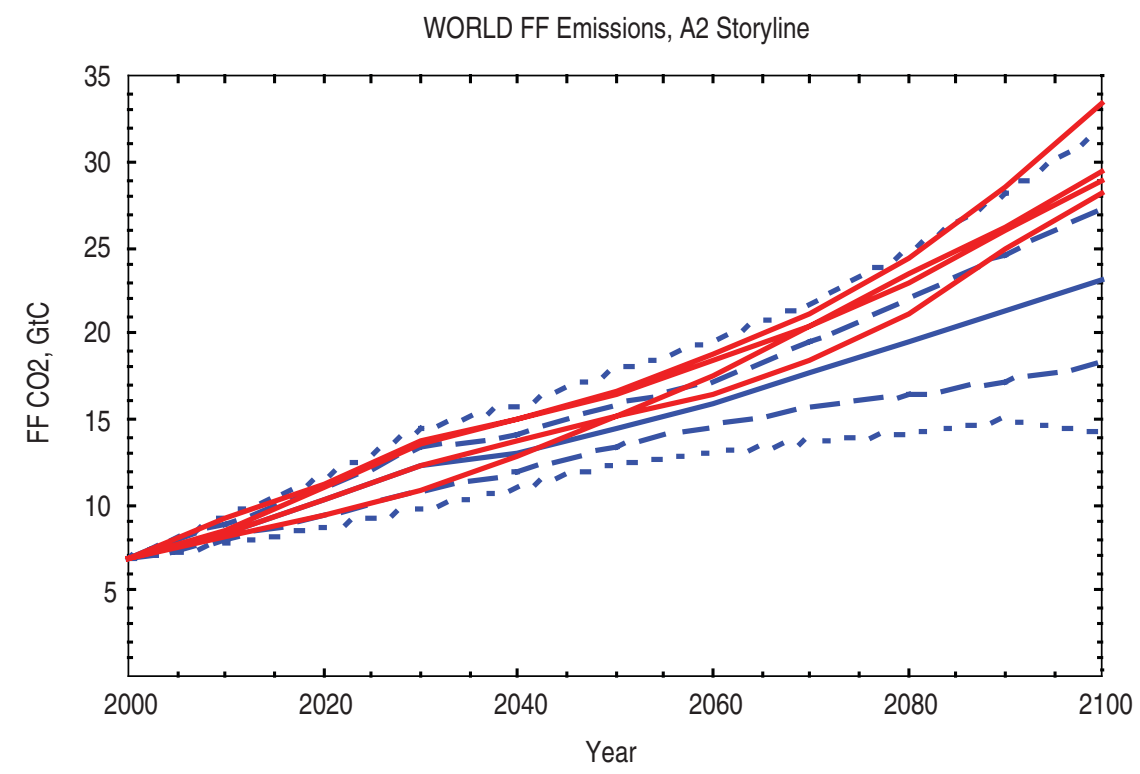

Figure 5 presents results for all storylines for the world and the two regions, reported in terms of cumulative $\mathrm{CO}_{2}$ emissions over the period 2000-2100 (cumulative emissions are a better indicator of impact on climate than emissions at a point in time). It shows that differences between the conditional projections and the SRES scenarios are the largest for the A2 storyline. In the B2 storyline, the SRES scenarios cover about half of the $95 \%$ uncertainty interval in the conditional projection, although they are still relatively high, with nearly all scenarios falling above the median of the conditional projections, particularly in the developing country region. For the A1 and B1 storylines the SRES scenarios essentially span the full $95 \%$ uncertainty interval of the conditional projections, both at the world level and within the two regions. This occurs even though the conditional probabilistic population projection for these storylines covers a wide range by 2100 , and is substantially lower than the single SRES population assumption in the industrialized country region (Figure 3b). The reason the SRES emissions scenarios compare differently to the conditional projections is that the SRES scenarios include a fairly wide range of possible per capita emissions trends in the A1 and B1 storylines (particularly in A1). Thus in terms of total emissions, uncertainty in per capita emissions can to some extent make up for a lack of uncertainty in population. This is not in general a good way to account for potential uncertainty in outcomes, however, because there are often other aspects of an analysis, besides total emissions, that may also depend on the population assumptions - land use change or climate change impacts are two examples. Uncertainty in per capita emissions will not make up for lack of uncertainty in population assumptions when addressing these other components of the problem. 
Figure 5. Cumulative $\mathrm{CO}_{2}$ emissions, 2000-2100, for individual SRES scenarios (gray dots), and percentiles (median and the bounds of the $60 \%$ and $95 \%$ uncertainty intervals) of conditional probabilistic projections, for each storyline.

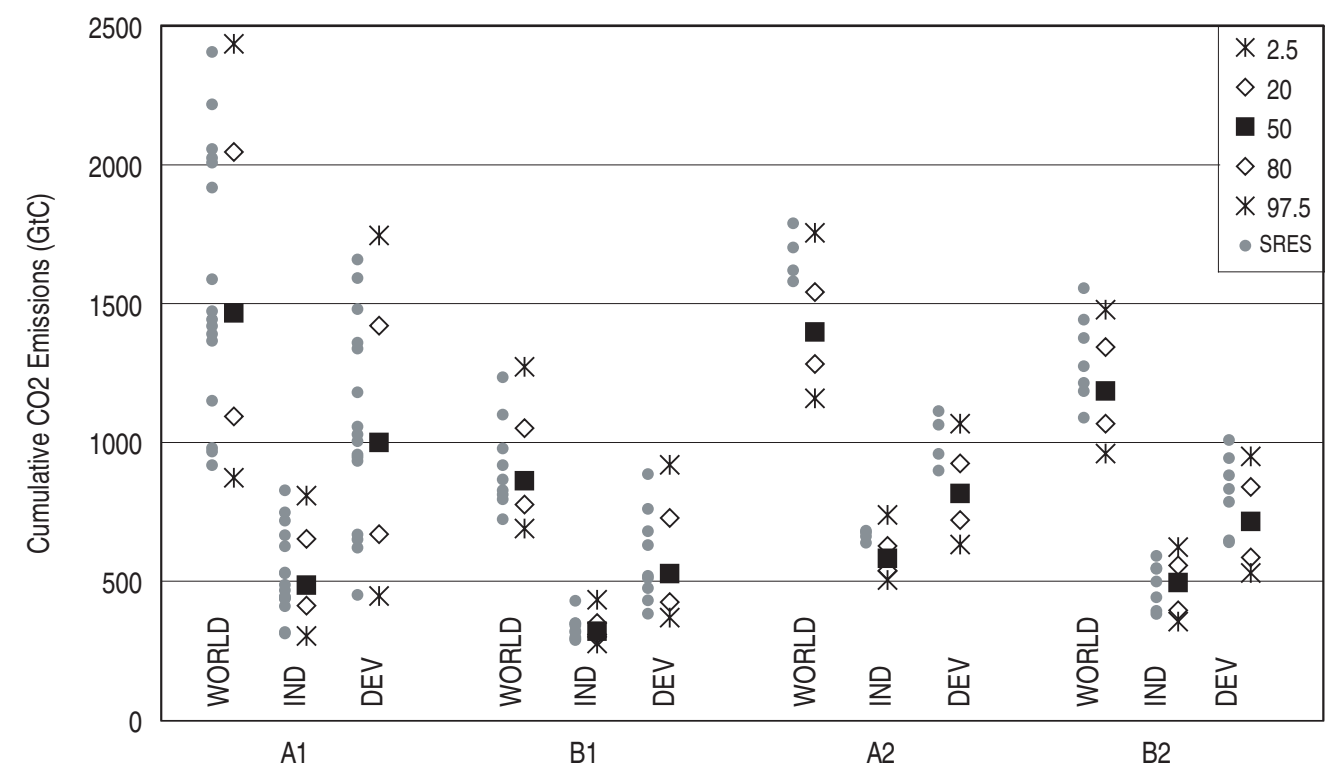

\section{Discussion and Conclusions}

Uncertainty in future greenhouse gas emissions has been approached in one of two ways: through the development and analysis of alternative scenarios, and through the fully probabilistic projections based largely on expert opinion. This paper has proposed a new option: the use of conditional probabilistic projections. This approach essentially combines the other two. Alternative sets of conditions are developed using scenario development methods, while uncertainty given a particular set of conditions is then defined probabilistically. The example presented here illustrated the concept using the SRES storylines as constraints in developing a set of four conditional probabilistic population and emissions projections.

Results demonstrate that the approach can provide a fuller accounting for uncertainty in population and emissions within storylines. They show, for example, that the uncertainty range for population within each storyline is wide, in contrast to the single population scenario assumed in SRES. In terms of emissions, the conditional probabilistic projections indicate an uncertainty distribution in the A2 and, to a lesser extent, the B2 storylines that is substantially wider than the range covered by the SRES scenarios. Furthermore, the conditional probabilistic approach can give a sense of whether existing emissions scenarios are biased in a particular direction. The B2 and, particularly, the A2 scenarios used in SRES fall at the upper end of the conditional distribution of emissions, suggesting that a fuller accounting for population uncertainty, using the most recent population projections, would produce generally lower emissions within these two storylines. These conclusions must remain tentative since they make important assumptions about relative likelihoods of per capita emissions paths and about relationships between variables. They are also dependent on the particular choices 
made in defining the criteria for the conditional projections. Nonetheless they suggest that substantial changes in the outlook for emissions (within storylines) are possible using the conditional probabilistic approach.

This exercise also raised several points regarding the population projections on which it is based. For example, the set of 1,000 simulations available for the IIASA projections (Lutz et al. 2001) contained relatively few simulations that matched a rather broad set of criteria for three variables and two world regions over one time period. Adding additional criteria, more regions, or additional time periods as constraints quickly makes the task impossible. This implies that when producing unconditional population projections, it would be useful to generate a much larger number of simulations in order to support applications calling for the conditional probabilistic approach. In addition, this application to the SRES scenarios demonstrated that the correlations assumed in the unconditional population projections have important implications for results. The IIASA projections are based on a single assumption about the strength of correlations between variables, over time, and across regions. This substantially reduces the number of simulations matching storylines that assume a different correlation. One effect is simply practical: it may be hard to find enough simulations in this case to characterize fully the uncertainty distribution. However it also means that the judgment of the relative likelihood across storylines, which should in principle be possible using the conditional probabilistic approach, is extremely sensitive to the specification of correlations. Conclusions regarding relative likelihoods must await further work on the empirical and theoretical basis for these correlations.

Finally, while the aim of this paper has been to explore how probabilistic approaches to uncertainty in population might benefit emissions projections, it is also worth considering the benefits of the storyline approach to the treatment of uncertainty in population projections. Storylines are a device for structuring thinking about a future with deep uncertainty. They are also a means of making projections more useful to users. Assumptions regarding the reasoning behind the choice of driving forces, parameter values, and modeling approaches are made more explicit. More explicit discussion of the reasoning motivating the input assumptions that underlie probabilistic population projections would make them more useful to the interdisciplinary research community. Demographers producing such projections might consider storylines as one way of communicating this information. Users from other fields need to know the conditions under which demographers think that, for example, fertility may turn out to be high in South Asia, or very low in Western Europe, or what might lead trends across regions to be strongly or weakly correlated. For a user searching for ways to join

projections from one field with projections from another, it is this underlying reasoning that provides the link. Making this reasoning as explicit as possible would greatly facilitate interdisciplinary work.

\section{References}

Alho, J. 1997. Scenarios, uncertainty and conditional forecasts of the world population. Journal of the Royal Statistical Society, Series A (Statistics in Society) 160(1): 71-85. 
Cubasch, U., G.A. Meehl, G.J. Boer, R.J. Stouffer, M. Dix, A. Noda, C.A. Senior, S. Raper, K.S. Yap, A. Abe-Ouchi, S. Brinkop, M. Claussen, M. Collins, J. Evans, I. Fischer-Bruns, G. Flato, J.C. Fyfe, A. Ganopolski, J.M. Gregory, Z.-Z. Hu, F. Joos, T. Knutson, R. Knutti, C. Landsea, L. Mearns, C. Milly, J.F.B. Mitchell, T. Nozawa, H. Paeth, J. Räisänen, R. Sausen, S. Smith, T. Stocker, A. Timmermann, U. Ulbrich, A. Weaver, J. Wegner, P. Whetton, T. Wigley, M. Winton, and F. Zwiers. 2001. Projections of future climate change. Pages 525582 (chapter 9) in J.T. Houghton, Y. Ding, D.J. Griggs, M. Noguer, P.J. van der Linden, X. Dai, K. Maskell, and C.A. Johnson (eds.), Climate Change 2001: The Scientific Basis. Contribution of Working Group I to the Third Assessment Report of the Intergovernmental Panel on Climate Change. Cambridge, U.K.: Cambridge University Press.

Dessai, S. and M. Hulme. 2001. Climatic implications of revised IPCC emissions scenarios, the Kyoto Protocol and quantification of uncertainties. Integrated Assessment 2: 159-170.

Dietz, T. and E.A. Rosa. 1997. Effects of population and affluence on $\mathrm{CO}_{2}$ emissions. Proceedings of the National Academy of Sciences USA 94: 175-179.

Edmonds, J., J. Reilly, R. Gardner, and A. Brenkert. 1986. Uncertainty in Future Global Energy Use and Fossil Fuel $\mathrm{CO}_{2}$ Emissions 1975 to 2075 (with appendices). Washington, D.C.: United States Department of Energy.

Gaffin, S.R. and B.C. O'Neill. 1997. Population and global warming with and without $\mathrm{CO}_{2}$ targets. Population and Environment 18(4): 389-413.

Grübler, A. and N. Nakicenovic. 2001. Identifying dangers in an uncertain climate. Nature 412(6842): 15.

Keepin, B. 1986. Review of global energy and carbon dioxide projections. Annual Review of Energy 11: 357-392.

Lempert, R.J., M.E. Schlesinger, S.C. Banks, and N.G. Andronova. 2000. The impacts of climate variability on near-term policy choices and the value of information. Climatic Change 45: 129-161.

Lutz, W., W. Sanderson, and S. Scherbov. 2001. The end of world population growth. Nature 412: 543-545.

Lutz, W. and S. Scherbov. 2002. Can immigration compensate for Europe's low fertility? Interim Report IR-02-052. Laxenburg, Austria: IIASA.

Lutz, W., W. Sanderson, S. Scherbov, and A. Goujon. 1996. World population scenarios for the $21^{\text {st }}$ century. Pages 361-396 in W. Lutz (ed.), The Future Population of the World. What Can We Assume Today? Revised Edition. London: Earthscan.

Moss, R.H. and S.H. Schneider. 2000. Uncertainties in the IPCC TAR: Recommendations to lead authors for more consistent assessment and reporting. In Cross Cutting Issues Guidance Papers. Geneva, Switzerland: Intergovernmental Panel on Climate Change.

Nakicenovic, N., J. Alcamo, G. Davis, B. de Vries, J. Fenhann, S. Gaffin, K. Gregory, A. Grübler, T.Y. Jung, T. Kram, E. Lebre La Rovere, L. Michaelis, S. Mori, T. 
Morita, W. Pepper, H. Pitcher, L. Price, K. Riahi, A. Roehrl, H.-H. Rogner, A. Sankovski, M. Schlesinger, P. Shukla, S. Smith, R. Swart, S. van Rooijen, N. Victor, and Z. Dadi. 2000. Special Report on Emissions Scenarios. Cambridge, U.K.: Cambridge University Press for the Intergovernmental Panel on Climate Change.

New, M. and M. Hulme. 2000. Representing uncertainty in climate change scenarios: A Monte Carlo approach. Integrated Assessment 1: 203-213.

Nordhaus, W.D. and D. Popp. 1997. What is the value of scientific knowledge? An application to global warming using the PRICE model. The Energy Journal 318: $1-45$.

Nordhaus, W.D. and G. Yohe. 1983. Future carbon dioxide emissions from fossil fuels. Cowles Foundation Paper No. 580. New Haven, CT: Yale University.

O'Neill, B.C., S. Scherbov, W. Lutz, and A. Goujon. Forthcoming. Population projections for the Millennium Assessment storylines. In preparation. Preliminary draft available at http://www.usf.uni-kassel.de/magmgroup/pop_new_3_round.htm.

O’Neill, B.C., F.L. MacKellar, and W. Lutz. 2001. Population and Climate Change. Cambridge, U.K.: Cambridge University Press.

Sanderson, W.C., S. Scherbov, W. Lutz, and B.C. O'Neill. Forthcoming. Applications of probabilistic population forecasting. In W. Lutz and W.C. Sanderson (eds.), The End of World Population Growth in the 21 ${ }^{\text {st }}$ Century: New Challenges for Human Capital Formation and Sustainable Development. London: Earthscan.

Sanderson, W.C., S. Scherbov, B.C. O’Neill, and W. Lutz. 2003. Conditional Probabilistic Population Forecasting. Interim Report IR-03-052. Laxenburg, Austria: IIASA.

Schneider, S.H. 2001. What is 'dangerous' climate change? Nature 411: 17-19.

Schneider, S.H. 2002. Can we estimate the likelihood of climatic changes at 2100 ? Climatic Change 52: 441-451.

Schwartz, P. 1991. The Art of the Long View. New York: Doubleday.

UN. 1998. World Population Projections to 2150. New York: United Nations.

Watson, R.T. and the Core Writing Team (35 authors). 2001. Climate Change 2001: Synthesis Report. Cambridge, U.K.: Cambridge University Press for the Intergovernmental Panel on Climate Change.

Webster, M.D., M. Babiker, M. Mayer, J.M. Reilly, J. Harnisch, M.C. Sarofim, and C. Wang. 2002. Uncertainty in emissions projections for climate models. Atmospheric Environment 36(22): 3659-3670.

Wigley, T.M.L. and S.C.B. Raper. 2001. Interpretation of high projections for globalmean warming. Science 293: 451-454. 